\title{
Natural history of caesarean scar pregnancy
}

\author{
Michał Pomorski, Tomasz Fuchs, Joanna Budny-Winska, \\ Aleksandra Zimmer, Mariusz Zimmer
}

2nd Department and Clinic of Gynaecology, Obstetrics and Neonatology, Wroclaw Medical University, Wroclaw, Poland

The rate of cesarean sections (CS) around the world is increasing at an 'alarming' rate. In Poland the rate of CS is 43.85\%. Increasing CS rates are associated with short-term and long-term maternal and perinatal consequences. Previous CS increases the risk of cesarean scar pregnancy (CSP), placenta previa, placenta accreta spectrum (PAS) disorders and

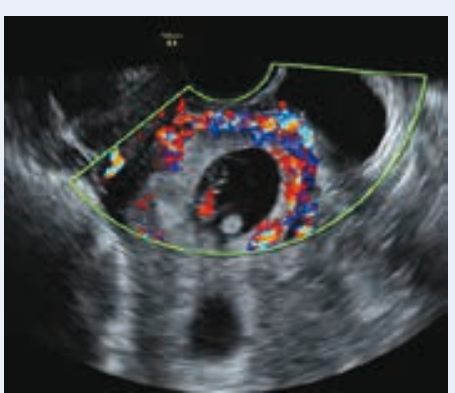

Figure 1. Cesarean scar pregnancy with early placenta accreta

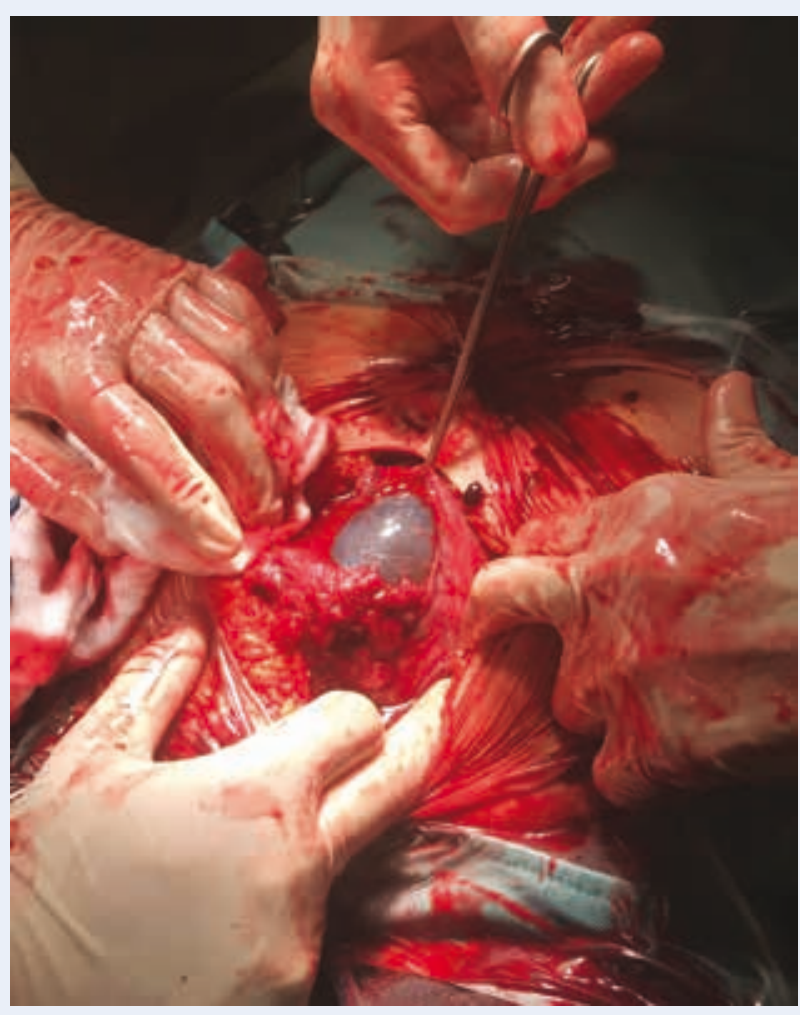

Figure 2. Rupture of the cesarean section scar a uterine rupture. According to the theory of professor Ilan Timor-Tritsch, placenta previa accreta in a woman after previous CS is a consequence of continuation of CSP that was not recognized or not treated in the first trimester.

A gravida 4 para 2 in the 8th gestational week (g.w.) was admitted to the Department of Gynecology and Obstetrics with suspected CSP. She had a history of one CS due to orthopedic indications and two missed abortions. The ultrasonographic (US) appearance revealed a gestational sac with the alive embryo (crown rump length $=8.5$ g.w.) surrounded by well vascularized trophoblastic tissue penetrating into the CS scar (Fig. 1). Thus, a CSP with early placenta accreta was diagnosed. The residual myometrial thickness (RMT) at the site of the CS scar was $1.5 \mathrm{~mm}$. The patient was offered an MTX injection into the gestational sac, but decided on the continuation of pregnancy being conscious of all of possible complications e.g.: a uterine rupture, a hemorrhage, a hysterectomy, the death of fetus and herself.

In 20 g.w., the US revealed the normal fetal growth and the normal amount of amniotic fluid. The placenta was diagnosed as complete previa, the sonographic features of PAS were present, including loss of retroplacental 'clear zone', multiple vascular lacunae ('Swiss cheese appearance') and 'bridging vessels'between the placenta and the bladder. The RMT value was $1 \mathrm{~mm}$. In 21 g.w., the patient was admitted to hospital with an abdominal pain and a hypovolemic shock. US appearance included an amniotic sac herniated through the prior CS scar, massive amount of fluid in the abdominal cavity and multiple hematomas around the uterus and next to the liver and the spleen. The patient was qualified to immediate laparotomy. After the removal of blood clots and around 1,500 mL of free blood, a uterine rupture (diameter $5 \mathrm{~cm}$ ) at the site of the $\mathrm{CS}$ scar with intensive arterial bleeding was shown (Fig. 2). After the extraction of the fetus, the placenta percreta was diagnosed with placental tissue penetrating into the bladder wall and into the parametrium. The persist-

Corresponding author:

Tomasz Fuchs

2nd Department and Clinic of Gynaecology, Obstetrics and Neonatology, Wroclaw Medical University, 213 Borowska St, 50-556 Wroclaw, Poland e-mail: tfuchs@02.pl 
ing hemorrhage and the patient's unstable cardiovascular status caused the need for urgent hemostasis. The presence of placenta percreta made a fast hysterectomy impossible, thus the Bakri balloon was inserted into the uterine cavity and, after the closure of the uterus, pumped with $500 \mathrm{~mL} 0.9 \%$ sodium chloride. The procedure was effective in controlling bleeding. After cardiovascular stabilization of the patient, the embolization of uterine arteries was performed. The Bakri-baloon was removed after 24 hours. No further bleeding was observed. After 10 days of recovery, the patient was discharged from hospital. 